\title{
MANEJO INTRAOPERATÓRIO DO SANGRAMENTO EM CIRURGIAS DE GRANDE PORTE COM MÉTODOS VISCOELÁSTICO
}

\author{
INTRAOPERATIVE MANAGEMENT OF BLEEDING IN MAJOR SURGERY \\ WITH VISCOELASTIC METHODS
}

Daniel Vieira de Queiroz, Marcelo Sampaio Duran, Aline Alonso Cherman

Study performed at Hospital Federal dos Servidores do Estado, Brazil.

Financial support: None.

Conflicts of interest: None.

Corresponding author: danielvieiradequeiroz@gmail.com

Submitted: jun 25; accepted after revision, oct 27, 2021.

\section{RESUMO}

A monitoração perioperatória da coagulação sanguínea é fundamental para estimar o risco de sangramento, diagnosticar coagulopatias, e orientar as terapias hemostáticas durante os procedimentos cirúrgicos. A avaliação da hemostasia e a escolha dos hemoderivados têm sido tradicionalmente feitas com base em testes convencionais de coagulação. No entanto, esses testes têm limitações, como tempos de resposta elevados e avaliação de apenas parte de todo o processo de formação do coágulo. Os testes viscoelásticos são uma alternativa à utilização dos métodos tradicionais de monitoramento da hemostasia, pois permitem uma avaliação global da coagulação sanguínea, desde o início da geração do trombo até sua dissolução, com resultados rápidos no atendimento. Dentre eles, está a tromboelastometria rotacional, que expressa graficamente a amplitude em função do tempo de todas as etapas de geração e lise do coágulo, traduzindo em valores absolutos os parâmetros analisados. As principais recomendações de uso desse método incluem o diagnóstico e orientação da terapia hemostática no período perioperatório de cirurgias de grande porte, como cirurgia cardíaca e transplante de fígado, e em quadros de politrauma e sangramento em pacientes críticos. Os objetivos deste estudo consistem em apresentar uma atualização dos métodos viscoelásticos (com foco na tromboelastometria rotacional) e sua aplicabilidade em diferentes cenários clínicos.

Descritores: tromboelastometria rotacional; coagulação; terapia guiada por metas; hemorragia aguda.

\section{ABSTRACT}

Perioperative monitoring of blood clotting is essential to estimate the risk of bleeding, to diagnose coagulopathy, and to guide hemostatic therapies during surgical 
Manejo intraoperatório do sangramento em cirurgias de grande porte com métodos viscoelástico

Queiroz DV, et al

procedures. Assessment of hemostasis and the choice of blood products have traditionally been made based on conventional coagulation tests. However, these tests have limitations, as they have high turnaround times and evaluate only the beginning of the whole clot formation process. Viscoelastic tests are an adequate alternative to the use of traditional methods of hemostasis monitoring, as they allow a global assessment of blood clotting, from the beginning of thrombus generation to its dissolution, with fast point-of-care results. Rotational thromboelastometry is a viscoelastic test that has gained importance in the perioperative setting. The test shows a graphical representation of clot formation in real-time and provides a range of parameters about the structure and kinetics of clot formation. The main recommendations of use of this method include diagnosing complex coagulopathy and guiding the hemostatic therapy in the perioperative setting of major surgery, such as cardiac surgery, liver transplant, major trauma, and bleeding in critical patients. The objective of this study is to present an update on viscoelastic methods (focusing on rotational thromboelastometry) and their applicability in different clinical scenarios.

Keywords: Thromboelastometry. Blood clotting. Early goal-guided therapy. Surgical blood loss.

\section{INTRODUÇÃO}

A identificação adequada e o tratamento precoce da causa específica de uma coagulopatia são fundamentais para o controle efetivo de um sangramento. O controle da hemorragia perioperatória deve ter por objetivo a racionalização das hemotransfusões, tendo como bases os exames e recursos disponíveis para diagnosticar a etiologia do sangramento e guiar a intervenção necessária ao seu controle, sempre se apoiando em evidências científicas. Um tratamento inadequado não apenas perpetua a perda sanguínea, mas também acarreta maior risco de ocorrência de eventos adversos e aumenta os custos com os cuidados de saúde.

Tradicionalmente, o uso de produtos do sangue tem sido indicado com base em testes convencionais da coagulação em associação com sinais clínicos de sangramento. Esses testes incluem, dentre outros: o tempo de atividade da protrombina (TAP) e a razão normalizada internacional (INR); o tempo de tromboplastina parcial ativada (PTTa); o tempo de trombina (TT); a dosagem de fibrinogênio sérico e a plaquetometria. No entanto, tais exames não foram validados para prever e/ou orientar a terapia da hemorragia aguda, e possuem algumas limitações como, por exemplo, avaliar somente a geração de trombina determinada pelos fatores pró-coagulantes e não considerar a interação desses fatores com os elementos figurados do sangue, já que são realizados em amostra de plasma (o que exige centrifugação do sangue total e aumenta o tempo até a obtenção do resultado) ${ }^{1}$. 
Manejo intraoperatório do sangramento em cirurgias de grande porte com métodos viscoelástico

Queiroz DV, et al

Já os métodos viscoelásticos, dentre os quais temos a tromboelastografia e a tromboelastometria rotacional, vêm sendo cada vez mais usados para a monitorização da coagulação e a tomada de decisão terapêutica acerca do uso de produtos do sangue e demais medicações hemostáticas. Por utilizarem o sangue total, esses ensaios conseguem abordar algumas lacunas deixadas pelos testes convencionais com resultados rápidos, demonstrando todo o processo desde a formação e estabilização do coágulo até sua dissolução, refletindo de forma mais fidedigna a hemostasia in vivo à beira do leito. Além disso, o uso de reagentes com aceleradores e inibidores permite identificar o distúrbio específico da coagulação apresentado pelo paciente, possibilitando o manejo adequado das alterações apresentadas. Dessa forma, tais testes tornaram-se ferramentas de grande interesse especialmente no contexto dinâmico do período intraoperatório, no qual a hemorragia geralmente tem etiologia multifatorial ${ }^{1}$. A presente revisão tem como objetivo discutir as principais aplicações clínicas dos testes viscoelásticos nas cirurgias de pacientes hepatopatas (incluindo transplante hepático), na cirurgia cardíaca e no tratamento de vítimas de politrauma.

\section{MANEJO DA COAGULAÇÃO NO TRANSPLANTE HEPÁTICO}

O fígado exerce papel central na manutenção da hemostasia, por ser o sítio de síntese da vasta maioria das proteínas requeridas para a regulação da coagulação e da fibrinólise. A manutenção da hemostasia normal requer um balanço entre o estímulo e a inibição à coagulação, essencial para garantir que não ocorra formação indesejada de trombos em condições fisiológicas. A doença hepática está associada a uma variedade de alterações capazes de romper o delicado equilíbrio entre formação e lise do coágulo, tais como a redução da síntese de fatores pró-coagulantes e anticoagulantes, a hiperfibrinólise, a trombocitopenia e a disfunção plaquetária.

Sendo o fígado a fonte da maioria dos fatores da coagulação, os níveis circulantes desses fatores podem ser significativamente reduzidos em pacientes com doença hepática crônica e lesão hepatocelular extensa. A redução nos fatores II, V, VII, IX, X e XI se correlaciona com a extensão da cirrose e a perda de função hepática. Já os níveis de fator VIII permanecem normais ou elevados, tanto pela produção extra-hepática (por exemplo, pelas células endoteliais) quanto pela depuração reduzida do complexo fator VIII-fator de von Willebrand pelo fígado. A redução da atividade do fator XIII se correlaciona diretamente com escores mais altos de Child-Pugh e MELD². Existe ainda uma tendência à redução dos níveis de fibrinogênio circulantes, proporcional ao grau de evolução da doença ${ }^{3}$. No entanto, o estado de estresse oxidativo ao qual o paciente cirrótico está submetido acarreta a oxidação das moléculas de fibrinogênio, provocando também alterações estruturais que levam à polimerização anormal dessas moléculas e à formação de redes de fibrina mais resistentes à lise, levando a um fenótipo prótrombótico 4 . 
Manejo intraoperatório do sangramento em cirurgias de grande porte com métodos viscoelástico

Queiroz DV, et al

É também no fígado que ocorre a geração da maioria dos inibidores da coagulação (antitrombina, proteína C, proteína S, cofator II da heparina e inibidor da via do fator tecidual) e dos componentes do sistema fibrinolítico (como plasminogênio e $\alpha_{2}$-antiplasmina), os quais são igualmente deficientes no indivíduo hepatopata ${ }^{5}$.

A hiperfibrinólise resulta de um desbalanço entre seus ativadores e inibidores. Os níveis de ativador do plasminogênio tecidual (tPA) estão aumentados pela depuração hepática reduzida, sem um crescimento proporcional dos níveis de inibidor do ativador do plasminogênio tipo 1 (PAI-1), levando o equilíbrio entre eles em direção à atividade excessiva do tPA. Além disso, o nível de atividade das proteínas antifibrinolíticas ( $\alpha_{2}$ antiplasmina e inibidor da fibrinólise ativado pela trombina) está reduzido na doença hepática, contribuindo para o aumento da fibrinólise. A hiperfibrinólise é encontrada em $30 \%$ a $50 \%$ dos pacientes com doença hepática crônica e se correlaciona diretamente com a gravidade da doença, de acordo com a classificação de Child-Pugh ${ }^{6}$.

Anormalidades em ambas a quantidade e a função plaquetárias são comuns na doença hepática crônica. Trombocitopenia é frequentemente observada na doença hepática avançada e os mecanismos que contribuem para ela incluem: sequestro esplênico (consequência da esplenomegalia secundária à hipertensão portal); redução da produção hepática de trombopoietina; supressão da medula óssea pelo vírus da hepatite $\mathrm{C}$ ou pelo tratamento antiviral; e aumento da destruição das plaquetas mediado por autoanticorpos e complexos imunes. Apesar disso, a atividade plaquetária geralmente é aumentada na doença hepática, já que normalmente há aumento do fator de von Willebrand (FvW) e redução da metaloproteinase ADAMTS13 (sintetizada pelo fígado) que limita a função do FvW, o que acaba por compensar o déficit quantitativo plaquetário 5 .

Por muito tempo acreditou-se que pacientes com doença hepática crônica e cirrose teriam tendência ao sangramento, estando protegidos do risco de trombose pelo fato de apresentarem alteração dos testes convencionais da coagulação (como TAP e PTTa). Entretanto, esses testes são mais sensíveis a alterações dos fatores prócoagulantes que dos anticoagulantes, superestimando a coagulopatia na doença hepática. Dessa forma, surgiu o conceito de "novo estado de equilíbrio" da hemostasia desses pacientes: a síntese deficiente de fatores pró-coagulantes é contrabalançada pela redução na síntese de fatores anticoagulantes e fibrinolíticos, assim como a trombocitopenia é contrabalançada pelo aumento da agregação causado pela maior atividade do FvW ${ }^{5}$. Sob circunstâncias normais, a hemostasia do paciente cirrótico se comporta essencialmente como a de um indivíduo sadio, ou seja, de forma equilibrada. No entanto, quando há exaustão dos mecanismos de compensação em situações de estresse, tanto sangramento difuso quanto trombose podem ocorrer ${ }^{5}$. A incidência de eventos trombóticos aumenta com a severidade da cirrose, sendo a trombose venosa profunda o evento mais comum nessa população, com uma prevalência de 2,1\% a 23,3\% em candidatos à transplante hepático sem hepatocarcinoma ${ }^{7}$. A hipercoagulabilidade se 
Manejo intraoperatório do sangramento em cirurgias de grande porte com métodos viscoelástico

Queiroz DV, et al

deve principalmente ao aumento do fator VIII combinado à redução da proteína C. A lentificação do fluxo sanguíneo portal e a lesão endotelial (relacionada ao estágio de evolução da doença hepática, à gravidade da hipertensão portal e à translocação bacteriana) contribuem para o desenvolvimento de trombose ${ }^{4}$.

Durante o transplante hepático, mudanças profundas no sistema hemostático podem ocorrer que se somam às alterações já existentes pela doença hepática: coagulopatia dilucional; hiperfibrinólise; coagulopatia induzida pelo trauma (devida a isquemia e lesão teciduais); e heparinização. Além disso, fenômenos trombóticos não são incomuns nessa cirurgia e aumentam a mortalidade. Tais fenômenos ocorrem principalmente em pacientes transplantados por doença colestática ou doença hepática gordurosa não-alcoólica ${ }^{5}$.

O transplante ortotópico do fígado (TOF) é dividido em três estágios: préanepático, anepático e neo-hepático, os quais apresentam peculiaridades no que diz respeito à hemostasia.

O estágio pré-anepático é aquele que vai da incisão cirúrgica até a exclusão vascular do fígado nativo. A maior parte do sangramento nessa fase é relacionada ao grau de dificuldade técnica da cirurgia ${ }^{8}$. 0 distúrbio hemostático presente nesse período geralmente se deve ao grau de disfunção hepática, por síntese e depuração reduzidas dos fatores de coagulação. Há aumento da atividade fibrinolítica pela redução da depuração do tPA enquanto os níveis de PAI-1 se mantem relativamente inalterados ${ }^{8}$.

O estágio anepático é aquele que vai da interrupção do fluxo sanguíneo para o fígado nativo até a reperfusão do enxerto. Nessa fase, as alterações da hemostasia se devem especialmente à redução da síntese dos fatores da coagulação e à hiperfibrinólise desencadeada pela falta de depuração do tPA ${ }^{8}$.

O estágio neo-hepático compreende o período desde a reperfusão do enxerto até o final da cirurgia. Nesse período, aparecem diversos distúrbios da hemostasia, por conta de heparinização (endógena, por dano ao glicocálice do endotélio vascular do enxerto, ou exógena, pela heparina administrada ao doador); redução global dos fatores da coagulação (função reduzida do novo fígado nesse primeiro momento) somada à coagulopatia dilucional, pela reposição volêmica associada à solução de preservação do enxerto; e aprisionamento de plaquetas nos sinusoides do enxerto. Ademais, alguns pacientes apresentam liberação acentuada de ativador de plasminogênio tecidural (tPA) do endotélio do enxerto, causando uma intensa atividade fibrinolítica ${ }^{8}$. 
Manejo intraoperatório do sangramento em cirurgias de grande porte com métodos viscoelástico

Queiroz DV, et al

\section{Métodos viscoelásticos no transplante hepático}

Ao contrário dos exames convencionais da coagulação, que, por limitações próprias, não refletem o verdadeiro estado de equilíbrio da hemostasia do paciente hepatopata, os testes viscoelásticos conseguem avaliar melhor o status hemostático desse paciente, suprindo parte das deficiências dos testes tradicionais e devendo ser associados a estes para guiarem as estratégias transfusionais no transplante hepático. A tromboelastometria rotacional mostrou-se promissora na avaliação e no tratamento de estados de hipercoagulação e hipocoagulação associados ao transplante hepático ${ }^{9}$.

Os exames mais usados para acompanhar a hemostasia na cirurgia hepática são os testes convencionais de laboratório e os testes feitos à beira do leito (tromboelastometria rotacional e tromboelastografia). Existem evidências que indicam que os métodos viscoelásticos podem predizer o risco de sangramento ou de trombose em pacientes submetidos a TOF, além de terem potencial de reduzir o sangramento, as transfusões e a mortalidade nos pacientes de alto risco ${ }^{5}$. A avaliação da presença de hiperfibrinólise durante o transplante idealmente deve ser feita com base em método viscoelástico, não sendo mais necessária hoje em dia a recomendação do uso profilático de antifibrinolíticos no TOF. Feito o diagnóstico de hiperfibrinólise, a utlização de drogas antifibrinolíticas pode reduzir significativamente $o$ sangramento e o uso de hemocomponentes ${ }^{5}$.

Sangramentos intraoperatório e pós-operatório são complicações maiores do transplante hepático e figuram entre as causas mais comuns de mortalidade após esse procedimento, especialmente no primeiro mês de pós-operatório ${ }^{10}$. Todavia, a transfusão de produtos do sangue durante cirurgia hepática também causa aumento da morbimortalidade. Após o TOF, taxas aumentadas de infecção e trombose de artéria hepática têm sido relacionadas à transfusão de concentrado de hemácias $(\mathrm{CH})$ - o risco de infecção aumenta em $7 \%$ por unidade de $\mathrm{CH}$ transfundida ${ }^{8}$. As transfusões de crioprecipitado, plasma fresco congelado (PFC) e concentrado de plaquetas (CP) demonstraram impacto negativo na sobrevida do enxerto em 1 e 5 anos e associação com lesão pulmonar aguda relacionada à transfusão (do inglês, TRALI) ${ }^{9}$. O uso de PFC em cirurgia hepática e o uso de CP no TOF foram associados a aumento de mortalidade 5 . O uso de algoritmo de transfusão baseado em TEG ou ROTEM pode guiar a administração desses produtos, evitando seu uso desnecessário. $O$ uso de concentrado de fibrinogênio para tratamento de hipofibrinogenemia guiado por ROTEM reduziu as taxas de transfusão de $\mathrm{CH}, \mathrm{PFC}$ e CP em mais de $50 \%$, além de ter aumentado a taxa de TOF sem transfusão desses produtos de $3,5 \%$ para $20 \%{ }^{5}$.

Dessa forma, o manejo perioperatório da coagulação usando estratégias restritivas de transfusão e algoritmos baseados em métodos viscoelásticos demonstrou benefícios na redução das necessidades de transfusão e melhora dos desfechos após o TOF. 
Manejo intraoperatório do sangramento em cirurgias de grande porte com métodos viscoelástico

Queiroz DV, et al

\section{MANEJO DA COAGULAÇÃO NA CIRURGIA CARDÍACA}

A cirurgia cardíaca com bypass cardiopulmonar é um procedimento com risco de perda sanguínea abundante e necessidade de hemotransfusão no perioperatório. 0 risco é influenciado, dentre outros fatores, pelo tipo e complexidade do procedimento, pela duração da circulação extracorpórea (CEC), pela técnica cirúrgica, pela história de cirurgia cardíaca prévia e pela existência de anormalidades hemostáticas préoperatórias. Sangramento excessivo foi descrito em 3\% a $10 \%$ dos pacientes submetidos à cirurgia cardíaca. Hemorragia pós-operatória e coagulopatia têm um grande impacto na morbimortalidade desse tipo de cirurgia, já que aumentam as taxas de transfusão de produtos do sangue, de reexploração cirúrgica e de eventos tromboembólicos, além de prolongarem os tempos de intubação e ventilação mecânica e de internação em unidade de terapia intensiva ${ }^{11}$. A cirurgia cardíaca é uma das classes de cirurgia que mais usa produtos do sangue, com uma taxa de $20 \%$ a $40 \%$ de transfusão nos procedimentos mais rotineiros $^{12}$.

A CEC prejudica o sistema hemostático, podendo levar a um sangramento excessivo. Primeiramente, as soluções utilizadas para o priming da CEC e para a cardioplegia provocam hemodiluição dos fatores de coagulação e elementos figurados do sangue que, devido à intensa ativação da hemostasia, são também altamente consumidos. A lesão tecidual pela cirurgia (com consequente exposição do fator tecidual das células endoteliais) e a ativação do fator XII pelo sangue exposto à superfície não endotelial do circuito de CEC ocasionam a ativação hemostática que tem potencial de gerar uma coagulopatia de consumo ${ }^{13}$.

Na cirurgia cardíaca, a anticoagulação é usada para evitar a formação de trombos no paciente e no circuito de CEC, e para minimizar a ativação excessiva do sistema hemostático e seus efeitos deletérios. Ainda assim, mesmo com as altas doses de heparina não fracionada utilizadas, ocorre ativação progressiva da coagulação e, consequentemente, da fibrinólise ${ }^{12}$. Hiperfibrinólise pode ser desencadeada pela ativação, mediada pela CEC, do fator XII, da calicreína e da trombina, pela hipotermia e pela liberação intravascular de fator tecidual e de tPA a partir das células endoteliais lesadas. A atividade elevada da plasmina pode levar à disfunção plaquetária (por reduzir a adesão e a agregação das mesmas) e até à hipofibrinogenemia (em média, a concentração de fibrinogênio reduz-se em $36 \%$ após a $C E C)^{12,14}$. A administração de ácido tranexâmico é capaz de reduzir as hemotransfusões, a perda sanguínea perioperatória e a taxa de reoperação, sem aumentar o risco de mortalidade ou eventos trombóticos ${ }^{12}$.

As altas doses de heparina podem contribuir para o aumento do sangramento após a saída do bypass, se forem neutralizadas inadequadamente pela protamina ou por recirculação da heparina sequestrada em proteínas ligadoras ou outros sítios. O "efeito rebote" da heparina consiste na recorrência da sua atividade anticoagulante mesmo após sua neutralização pela protamina, e tem potencial de inibir a coagulação e a função 
Manejo intraoperatório do sangramento em cirurgias de grande porte com métodos viscoelástico

Queiroz DV, et al

plaquetária. Entretanto, o excesso de protamina pode ter efeito adverso semelhante, além de potencializar a fibrinólise e causar trombocitopenia ${ }^{12,13,15}$.

As plaquetas também são afetadas pela CEC, com redução do seu número e função, principalmente no que tange à agregação plaquetária. Essa alteração deve-se à hemodiluição e ao consumo provocado por destruição mecânica e adesão ao circuito de CEC. Estudos mostram redução da contagem plaquetária em $45 \%$ e da função de $54 \%$ a $71 \%$ (nos testes de agregometria plaquetária). A normalização da função pode levar de 3 a 6 horas após a CEC ${ }^{12,13}$. A manutenção de altas concentrações séricas da heparina na CEC prolongada e o uso de antifibrinolíticos ajudam a preservar a função plaquetária, já que ocorre menor ativação das mesmas e menor formação de plasmina ${ }^{14}$.

A hipotermia, usada na cirurgia cardíaca com CEC com o objetivo principal de propiciar proteção orgânica contra a isquemia, pode levar à coagulopatia de diferentes formas. Ela tem o potencial de induzir alterações morfológicas nas plaquetas causando sua disfunção ${ }^{16}$, especialmente quando há exposição do sangue hipotérmico a um agente ativador, como o circuito da CEC. A hipotermia, por retardar a atividade enzimática no geral, também reduz a função dos fatores de coagulação ${ }^{16}$. Ainda, por gerar lesão endotelial com subsequente liberação de fator tecidual e estimulação da formação de trombina, a hipotermia é capaz de induzir a fibrinólise ${ }^{17,18}$.

\section{Métodos viscoelásticos na cirurgia cardíaca}

O tratamento da coagulopatia relacionada ao uso da CEC na cirurgia cardíaca é complexo, devido à sua natureza multifatorial. O uso empírico de produtos do sangue para tratar e tentar distinguir sangramento microvascular de sangramento cirúrgico está longe de ser uma boa estratégia para o manejo desse distúrbio. O uso dos testes convencionais da coagulação, apesar de oferecer uma base racional para diagnóstico e tratamento do sangramento, tem sua utilidade limitada pelo longo tempo requerido para a obtenção dos resultados, além de outros entraves, como a impossibilidade de realização dos testes de TAP e PTTa por causa do uso de heparina em altas doses durante a CEC 11,12 .

No entanto, o uso de testes à beira do leito permite um manejo mais adequado do sangramento, de acordo com as anormalidades apresentadas pelo sistema hemostático, pautando a decisão terapêutica. Esses testes possibilitam a diferenciação entre sangramento microvascular e cirúrgico e a administração da terapia hemostática específica de forma mais precoce. $\mathrm{O}$ uso de testes viscoelásticos à beira do leito demonstrou reduzir transfusões em até $50 \%$ e melhorar o desfecho dos pacientes, em comparação ao manejo com testes convencionais ${ }^{12}$.

A implementação de algoritmos de transfusão na cirurgia cardíaca com base em em testes viscoelásticos aparenta ser superior ao uso de algoritmos baseados nos testes tradicionais ${ }^{11}$. O emprego de um protocolo de manejo do sangramento na cirurgia 
Manejo intraoperatório do sangramento em cirurgias de grande porte com métodos viscoelástico

Queiroz DV, et al

cardíaca guiado por tromboelastometria permitiu diminuir a quantidade de produtos do sangue usados e o número de pacientes que demandam transfusão durante as cirurgias. Estudos sugerem que a utilização de tais protocolos reduz o sangramento perioperatório, o tempo de internação e a morbimortalidade, além de impactar nos custos com o tratamento - estudo do coorte prospectivo publicado em 2019 por Kuiper e cols. sugeriu uma economia potencial de 4800 euros por paciente ${ }^{11,12,19,20}$.

A possibilidade de uso de um instrumento à beira do leito facilita o monitoramento da concentração circulante de heparina e dos seus efeitos anticoagulantes. Classicamente, a administração de heparina e de protamina, para sua reversão, é feita com base nos resultados de tempo de coagulação ativado (TCA) coletados de forma seriada durante o procedimento cirúrgico. Contudo, há evidências de que o TCA após administração da protamina não reflete as concentrações de heparina residual de forma acurada e não deve ser usado para titular dose de protamina ${ }^{15}$. Estudo in vitro demonstrou que o TCA aumenta quanto maior a dose de protamina em relação à heparina. Também se observou que um TCA prolongado após neutralização da heparina com protamina em razão de 1:1 não possui valor preditivo para detectar efeito residual de heparina. Outros estudos mostraram a ausência de relação linear entre os valores de TCA e de heparina. Já os ensaios INTEM e HEPTEM permitem avaliação específica da heparina residual em cirurgias cardíacas ${ }^{15,20}$, apesar do INTEM também ter demonstrado sensibilidade à protamina, especialmente quanto maior a relação protamina/heparina.

O uso do método viscoelástico também pode ser estendido ao pós-operatório da cirurgia cardíaca. O sangramento no período pós-cirúrgico é uma das complicações mais comuns desse tipo de cirurgia, chegando a aproximadamente $20 \%$ de sangramentos significativos, havendo necessidade de reexploração cirúrgica em $5 \%$ dos pacientes. Pelo menos metade dos pacientes submetidos à reoperação têm, de fato, uma causa cirúrgica de sangramento, enquanto, no restante, a causa do sangramento é multifatorial e relacionada às circunstâncias particulares ao procedimento ${ }^{12,21}$. O uso da tromboelastometria rotacional permite diferenciar o sangramento de origem cirúrgica de outros tipos de sangramento, evitando reabordagens desnecessárias que aumentam a morbimortalidade ${ }^{11}$, e direcionando a terapia de controle do sangramento pósoperatório.

Em resumo, a utilização de algoritmos norteados por métodos viscoelásticos em cirurgia cardíaca permite reduzir a perda sanguínea e o número de produtos do sangue transfundidos no perioperatório. A implementação do método tem o potencial de reduzir grandemente os custos financeiros associados a esse tipo de procedimento, demonstrando a custo-efetividade de sua utilização. 
Manejo intraoperatório do sangramento em cirurgias de grande porte com métodos viscoelástico

Queiroz DV, et al

\section{MANEJO DA COAGULAÇÃO NO TRAUMA}

Os métodos viscoelásticos possuem uma utilidade muito bem documentada dentro do centro cirúrgico, na cirurgia cardíaca com CEC e na cirurgia hepática, por exemplo. Todavia, existe uma crescente tendência de expandir suas aplicações para fora da sala de cirurgia, especialmente para conduzir o tratamento da coagulopatia induzida pelo trauma $(\mathrm{CIT})^{20}$, uma condição que envolve desordens da coagulação e inflamação, caracterizada por prejuízos na formação e lise do coágulo e na homeostasia vascular no geral $^{22}$.

Em pacientes de trauma grave, uma perda sanguínea significativa é frequentemente associada à coagulopatia ${ }^{23}$ que ocorre em um quarto a um terço desses pacientes 22,24 . A hemorragia não controlada continua sendo a causa mais comum de morte prevenível após o trauma e é responsável por aproximadamente metade desses óbitos ${ }^{24}$, apesar da implementação difundida dos princípios do controle de danos e dos avanços no cuidado ao paciente vítima de trauma. Todavia, a morte por hemorragia não é a única complicação da CIT. Devido às interseções entre as vias da coagulação e da inflamação, uma série de eventos adversos pode se associar à coagulopatia do trauma, incluindo complicações tromboembólicas e disfunção orgânica ${ }^{22,25}$.

A etiologia da CIT envolve ativação intensa do fator tecidual, hipoperfusão, inativação de fatores da coagulação (V e VIII) pela proteína C ativada e hiperfibrinólise, que levam à formação deficiente de coágulos. Já o agravamento da CIT se dá por consumo de fatores da coagulação e plaquetas, disfunção plaquetária induzida pelo trauma, anemia, hemodiluição, transfusões maciças, hipotermia e acidose metabólica (os dois últimos associados à coagulopatia formam a chamada "tríade letal"). A capacidade de detectar e tratar esse fenômeno de forma precoce parece ser uma peçachave no controle da hemorragia do paciente vítima de evento traumático ${ }^{22,25}$.

A resposta endócrino-metabólica ao trauma leva à liberação de catecolaminas na corrente sanguínea, causando lesão endotelial e degradação do glicocálice. Os altos níveis de adrenalina e a endoteliopatia se associam à hipocoagulabilidade e à hiperfibrinólise, contribuindo para a ocorrência da CIT. Ainda, o escape de componentes do glicocálice para a circulação contribui para a autoheparinização observada em aproximadamente $5 \%$ dos pacientes de trauma ${ }^{22,24,25}$. A hipoperfusão tecidual ativa a via de contato da coagulação, estimula a ativação da proteína C e agrava a lesão tecidual, o que exacerba a resposta inflamatória e pode culminar em disfunção orgânica múltipla 22 .

A reposição volêmica após hemorragia maciça no trauma pode levar à intensa diluição dos fatores da coagulação, resultando em coagulopatia dilucional (também chamada de coagulopatia iatrogênica), cuja incidência cresce quanto maior a quantidade administrada de fluidos ${ }^{25}$. A concentração plasmática de fibrinogênio e a plaquetometria rapidamente caem, de forma proporcional à extensão da hemodiluição. 
Manejo intraoperatório do sangramento em cirurgias de grande porte com métodos viscoelástico

Queiroz DV, et al

Níveis adequados de fibrinogenemia são essenciais para o manejo da coagulopatia dilucional, na medida em que estudos mostram que baixos níveis de fibrinogênio se associam a piores desfechos, como maior necessidade transfusional e morte 24,25 .

Durante a reanimação volêmica, também pode ser observada a ocorrência de hipotermia e acidose, que levam à redução da atividade dos fatores de coagulação, lentificando o processo de formação e propagação do coágulo. Além disso, a hipotermia reduz a função plaquetária e a acidose favorece a degradação da fibrina, levando ao maior consumo e consequente queda dos níveis plasmáticos de fibrinogênio ${ }^{22,25}$. A correção da acidose com bicarbonato não está associada à reversão da coagulopatia ${ }^{25}$.

Fibrinólise precoce associada a ressangramento pode ocorrer após hemodiluição excessiva, pela diminuição da concentração e da interação das proteínas antifibrinolíticas endógenas, e é também estimulada pelos altos níveis de proteína $C$ ativada circulantes ${ }^{22,24}$. A hiperfibrinólise se mostrou associada ao aumento da mortalidade e das transfusões maciças no trauma ${ }^{22}$. O uso profilático de ácido tranexâmico, desde que iniciado nas primeiras 3 horas do trauma, mostrou-se efetivo na redução da mortalidade geral, sem aumento aparente do risco de eventos adversos vaso-oclusivos, como demonstrado pelo estudo CRASH-2 ${ }^{24,25,26}$. Mais recentemente, descreveu-se o fenômeno do "colapso fibrinolítico" ("fibrinolytic shutdown") no trauma, que seria mais comum que a hiperfibrinólise, apesar de causar menor mortalidade que esta (34\% de mortalidade da hiperfibrinólise contra 22\% do "colapso") 22,24.

A coagulopatia compele a uma estratégia precoce e rápida de tratamento hemostático para prevenir a exsanguinação. Muitos centros de trauma usam protocolos de transfusão maciça baseados em relações empíricas entre os $\mathrm{CH}$, PFC e CP administrados, por exemplo, na proporção 1:1:1. Todavia, o ponto a partir do qual a administração de mais PFC e CP (para manter a razão com os $\mathrm{CH}$ administrados) passa a ser excessiva é incerto ${ }^{27}$, o que constitui um problema frente aos riscos associados ao uso desses produtos. Estudos com pacientes vítimas de trauma e choque hemorrágico já demonstraram que o uso de PFC foi associado, de forma independente, à maior incidência de falência de múltiplos órgãos e síndrome do desconforto respiratório agudo (SDRA $)^{1}$. Ainda, o PFC agrava a questão da hemodiluição, possui baixos níveis de fibrinogênio (insuficientes para corrigir a deficiência desse fator) ${ }^{23,28}$ e é ineficiente para correção da coagulopatia ${ }^{25}$, com estudo demonstrando que o PFC só consegue melhorar a formação do coágulo quando administrado em conjunto com crioprecipitado e $\mathrm{CP}^{28}$. Já o uso de CP (independente da contagem plaquetária) como parte de tais protocolos de reanimação balanceada não apenas é incapaz de reverter os distúrbios da agregação plaquetária induzidos pelo trauma, mas também causa aumento da morbimortalidade, conforme demonstrado em múltiplos estudos ${ }^{22,25}$.

Como opção à reanimação volêmica hemostática baseada no modelo de "controle de danos" (com protocolos de transfusão que apresentam relações empíricas 
Manejo intraoperatório do sangramento em cirurgias de grande porte com métodos viscoelástico

Queiroz DV, et al

entre os produtos do sangue), pode-se usar a terapia hemostática guiada por testes viscoelásticos.

\section{Métodos viscoelásticos no trauma}

Os testes convencionais da coagulação, apesar de muito usados na avaliação de anormalidades da coagulação em cenários de trauma e transfusão maciça, apresentam algumas limitações nessas circunstâncias, como por exemplo a incapacidade de detectar um estado hiperfibrinolítico ou de avaliar disfunção plaquetária. Já o uso de um teste viscoelástico como a tromboelastometria rotacional permite detectar as perturbações da coagulação sistêmica e conduzir a terapia hemostática dos pacientes vítimas de politraumatismo com rapidez e de acordo com as necessidades apresentadas, o que é essencial para reverter a patofisiologia da CIT e melhorar os desfechos.

O diagnóstico de $\mathrm{CIT}$ por testes convencionais geralmente é feito através do alargamento de TAP/INR e PTTa. Diversos estudos comparando o INR com os testes viscoelásticos no trauma mostraram que estes possuem alguns parâmetros (como A5, A10 e A15) com boa sensibilidade e especificidade para avaliação da $\mathrm{CIT}^{28}$. Outro parâmetro usado para diagnóstico da CIT é a dosagem quantitativa de fibrinogênio, cujo padrão-ouro é a dosagem através do ensaio de Clauss. Estudos mostram que o diagnóstico de deficiência de fibrinogênio através dos métodos viscoelásticos tem boa correlação com o diagnóstico pelo método de Clauss, com a vantagem da obtenção dos resultados em muito menos tempo ${ }^{28,29}$. Além disso, os ensaios viscoelásticos são capazes de identificar a auto-heparinização associada à degradação do glicocálice nas vítimas de trauma ${ }^{22}$. Assim, comparados aos testes convencionais, os tetes viscoelásticos parecem diagnosticar a CIT com maior precisão ${ }^{28}$, além de o fazerem mais rapidamente.

A descoberta do fenômeno do "colapso fibrinolítico" trouxe uma nova dimensão acerca do uso de antifibrinolíticos e dos métodos viscoelásticos no trauma, já que estes são a única forma de detectar a fibrinólise de forma acurada e à beira do leito. 0 conceito do "shutdown" fala a favor da administração seletiva do ácido tranexâmico no trauma, respaldada pela redução na mortalidade observada com o uso dessa medicação guiado por metas $22,29,30$.

A grande aplicação clínica de um teste viscoelástico no trauma é no uso de protocolos de transfusão maciça guiada. Com a utilização crescente desse tipo de protocolo, a ocorrência da coagulopatia iatrogênica vem caindo, já que se evita a administração desnecessária de volumes vultosos de fluidos ${ }^{22}$. Além disso, frente à complexa patofisiologia da CIT, um tratamento personalizado, orientado para as necessidades de cada paciente, se torna de suma importância ${ }^{30}$.

Gonzalez e cols. ${ }^{27}$ publicaram um ensaio clínico randomizado comparando a transfusão guiada por métodos viscoelásticos à transfusão guiada por testes convencionais em 111 pacientes adultos com hemorragia secundária a trauma. $O$ estudo 
Manejo intraoperatório do sangramento em cirurgias de grande porte com métodos viscoelástico

Queiroz DV, et al

demonstrou maior sobrevida do grupo transfusão guiada por teste viscoelástico, além de menor uso de PFC, CP e crioprecipitado nesse grupo na fase inicial do trauma, sugerindo que um tratamento baseado em protocolo de transfusão maciça guiado por método viscoelástico permite o uso apropriado de produtos do sangue, evitando tanto a subutilização quanto o sobreuso. Também foi observado que este grupo apresentou menores tempos de internação em Unidade de Terapia Intensiva e de ventilação mecânica.

Os benefícios do uso do método viscoelástico no manejo do paciente de trauma grave se estendem também aos pacientes pediátricos. Deng e cols. ${ }^{23}$, em uma coorte multicêntrica retrospectiva comparando 332 crianças vítimas de trauma tratadas com terapia guiada por tromboelastometrial rotacional e 332 tratadas com base nos testes convencionais, evidenciaram que o tempo até o início da administração de produtos do sangue, o tempo de internação e o uso de PFC foram menores no grupo guiado por tromboelastometrial rotacional, destacando os benefícios do uso desse método na população pediátrica vítima de trauma.

Considerando que a fisiopatologia da coagulopatia envolve polimerização deficiente do fibrinogênio, hiperfibrinólise e lentificação na iniciação do coágulo (dados que podem ser negligenciados pelos testes convencionais), os ensaios viscoelásticos aparentam ser mais apropriados que os testes tradicionais no diagnóstico e tratamento da CIT em traumas agudos. Apesar de aceitável em um contexto de risco de morte por hemorragia grave, a estratégia de reanimação volêmica hemostática no modelo de controle de danos apresenta todos os riscos associados à transfusão maciça, devendo, sempre que possível, ser preterida em relação à estratégia guiada por teste viscoelástico.

\section{CONCLUSÃO}

As desordens da hemostasia são anomalias que provocam um aumento da morbimortalidade cirúrgica. O manejo inadequado dessas desordens com uso empírico de produtos hemostáticos e protocolos de transfusão maciça pode levar ao agravamento da coagulopatia e a várias complicações associadas ao uso desses elementos.

O monitoramento da hemostasia no período perioperatório pode ser feito com base em diferentes tipos de métodos. Os testes viscoelásticos apresentam consideráveis vantagens sobre os métodos tradicionalmente utilizados, como a possibilidade de oferecerem resultados rápidos, à beira do leito, de fácil interpretação e com uma visão global sobre todas as etapas da dinâmica de formação do coágulo. Eles constituem ferramentas valiosas para o diagnóstico e tratamento de coagulopatias em diversos contextos clínicos, incluindo cirurgias cardíaca, hepática e de grande porte no geral, 
Manejo intraoperatório do sangramento em cirurgias de grande porte com métodos viscoelástico

Queiroz DV, et al

hemorragias secundárias ao trauma, além da avaliação de estados de hipercoagulabilidade.

Desse modo, considerando que os métodos viscoelásticos permitem uma intervenção terapêutica rápida e dirigida à disfunção apresentada, racionalizando hemotransfusões e otimizando o tratamento de pacientes graves com sangramento ativo de forma segura e custo-efetiva, faz-se imprescindível que o conhecimento das bases técnicas e princípios desses testes sejam difundidos na prática médica, devendo ser cada vez mais aplicados de rotina nos protocolos de tratamento de distúrbios hemostáticos.

\section{REFERÊNCIAS}

1. Crochemore T, Piza FMT, Rodrigues RR, Guerra JCC, Ferraz LR, Corrêa TD. A nova era da tromboelastometria. Einstein. 2017 Jun;15(3):380-5.

2. Bedreli S, Sowa JP, Malek S, Blomeyer S, Katsounas A, Gerken G, et al. Rotational thromboelastometry can detect factor XIII deficiency and bleeding diathesis in patients with cirrhosis. Liver Int. 2017 Apr;37(4):562-8.

3. Li CJ, Yang ZH, Lu FG, Shi XL, Liu DL. Clinical significance of fibrotic, haemostatic and endotoxic changes in patients with liver cirrhosis. Acta Gastroenterol Belg. Jul-Sep 2018;81(3):404-9.

4. Becatti M, Mannucci A, Argento FR, Gitto S, Vizzutti F, Marra F, et al. Superresolution microscopy reveals an altered fibrin network in cirrhosis: the key role of oxidative stress in fibrinogen structural modifications. Antioxidants. 2020 Aug 12;9(8):737.

5. Hartmann M, Szalai C, Saner FH. Hemostasis in liver transplantation: pathophysiology, monitoring, and treatment. World J Gastroenterol. 2016 Jan 28;22(4):1541-50.

6. Leebeek FWG, Rijken DC. The fibrinolytic status in liver diseases. Semin Thromb Hemost. 2015 Jul;41(5):474-80.

7. European Association for the Study of the Liver. EASL clinical practice guidelines: vascular diseases of the liver. J Hepatol. 2016 Jan;64(1):179-202.

8. Clevenger B, Mallett SV. Transfusion and coagulation management in liver transplantation. World J Gastroenterol. 2014 May 28;20(20):6146-58.

9. Zamper RPC, Amorim TC, Costa LGV, Takaoka F, Neto AS. O papel da tromboelastometria na avaliação e no tratamento da coagulopatia em pacientes submetidos ao transplante hepático. Einstein. 2017 Apr 04;15(2):243-6.

10. Baganate F, Beal EW, Tumin D, Azoulay D, Mumtaz K, Black SM, et al. Early mortality after liver transplantation: defining the course and the cause. Surgery. 2018 Oct;164(4):694-704.

11. Bolliger D, Tanaka KA. Point-of-care coagulation testing in cardiac surgery. Semin Thromb Hemost. 2017 Jun;43(4):386-96. 
Manejo intraoperatório do sangramento em cirurgias de grande porte com métodos viscoelástico

Queiroz DV, et al

12. Bartoszko J, Karkouti K. Managing the coagulopathy associated with cardiopulmonary bypass. J Thromb Haemost. 2021 Mar;19(3):617-32.

13. Fang ZA, Navaei AH, Hensch L, Hui SKR, Teruya J. Hemostatic management of extracorporeal circuits including cardiopulmonary bypass and extracorporeal membrane oxygenation. Semin Thromb Hemost. 2020 Feb;46(1):62-72.

14. Earnshaw C, Poole M. Tranexamic acid. Anaesth Tut Week. 2019 Jul 09;(406):1-7.

15. Boer C, Meesters MI, Veerhoek D, Vonk ABA. Anticoagulant and side-effects of protamine in cardiac surgery: a narrative review. $\mathrm{Br} J$ Anaesth. 2018 May;120(5):914-27.

16. Horioka K, Tanaka H, Isozaki S, Okuda K, Asari M, Shiono H, et al. Hypothermiainduced activation of the splenic platelet pool as a risk factor for thrombotic disease in a mouse model. J Thromb Haemost. 2019 Oct;17(10)1762-71.

17. Sousa RL, Ferreira LC, Garcia FL, Franco LHM, Alves LL. Plasma fresco congelado, plaquetas e crioprecipitado: quando e como usar. Rev Med Minas Gerais. 2014;24 Suppl 8:S81-6.

18. Ise H, Kitahara H, Oyama K, Takahashi K, Kanda H, Fujii S, et al. Hypothermic circulatory arrest induced coagulopathy: rotational thromboelastometry analysis. Gen Thorac Cardiovasc Surg. 2020 Aug;68(8):754-61.

19. Kuiper GJAJM, Van Egmond LT, Henskens YMC, Roekaerts PM, Maessen JG, Ten Cate $\mathrm{H}$, et al. Shifts of transfusion demand in cardiac surgery after implementation of rotational thromboelastometry-guided transfusion protocols: analysis of the HEROES-CS (HEmostasis Registry of patiEntS in Cardiac Surgery) observational, prospective open cohort database. J Cardiothorac Vasc Anesth. 2019 Feb;33(2):30717.

20. Junior HCR, Zon Filippi AC. Aspectos técnicos e aplicações clínicas de testes viscoelásticos no monitoramento da hemostasia perioperatória. Rev Saúde. 2020 Jan/Jun;11(1):29-35.

21. Choi YJ, Yoon SZ, Joo BJ, Lee JM, Jeon YS, Lim YJ, et al. A perda sanguínea excessiva no pós-operatório de cirurgia cardíaca pode ser prevista com o sistema de classificação da Sociedade Internacional de Trombose e Hemostasia (ISTH). Rev Bras Anestesiol. 2017;67(5):508-15.

22. Kornblith LZ, Moore HB, Cohen MJ. Trauma-induced coagulopathy: the past, present, and future. J Thromb Haemost. 2019 Jun;17(6):852-62.

23. Deng Q, Hao F, Wang Y, Guo C. Rotation thromboelastometry (ROTEM) enables improved outcomes in the pediatric trauma population. J Int Med Res. 2018 Dec;46(12):5195-204.

24. Chang R, Cardenas JC, Wade CE, Holcomb JB. Advances in the understanding of trauma-induced coagulopathy. Blood. 2016 Aug 25;128(8):1043-9.

25. Petros S. Trauma-induced coagulopathy. Hamostaseologie. 2019 Feb;39(1):20-27.

26. Roberts I, Shakur H, Coats T, Hunt B, Balogun E, Barnetson L, et al. The CRASH-2 trial: a randomised controlled trial and economic evaluation of the effects of tranexamic 
Manejo intraoperatório do sangramento em cirurgias de grande porte com métodos viscoelástico

Queiroz DV, et al

acid on death, vascular occlusive events and transfusion requirement in bleeding trauma patients. Health Technol Assess. 2013 Mar;17(10):1-79.

27. Gonzalez E, Moore EE, Moore HB, Chapman MP, Chin TL, Ghasabyan A, et al. Goaldirected hemostatic resuscitation of trauma-induced coagulopathy: a pragmatic randomized clinical trial comparing a viscoelastic assay to conventional coagulation assays. Ann Surg. 2016 Jun;263(6):1051-9.

28. Wirtz MR, Baumann HM, Klinkspoor JH, Goslings JC, Juffermans NP. Viscoelastic testing in trauma. Semin Thromb Hemost. 2017 Jun;43(4):375-85.

29. Hartmann J, Walsh M, Grisoli A, Thomas AV, Shariff F, McCauley R, et al. Diagnosis and treatment of trauma-induced coagulopathy by viscoelastography. Semin Thromb Hemost. 2020 Mar;46(2):134-46.

30. Gonzalez E, Moore EE, Moore HB. Management of trauma-induced coagulopathy with thromboelastography. Crit Care Clin. 2017 Jan;33(1):119-34. 